\title{
A Brief Overview of the WHO Classification of Breast Tumors, 4th Edition, Focusing on Issues and Updates from the 3rd Edition
}

\author{
Hans-Peter Sinn ${ }^{\mathrm{a}}$ Hans Kreipe ${ }^{\mathrm{b}}$ \\ aSektion für Gynäkologische Pathologie, Pathologisches Institut, Ruprecht-Karls-Universität Heidelberg, \\ ${ }^{b}$ Institut für Pathologie, Medizinische Hochschule Hannover, Germany
}

\section{Keywords \\ Diagnostic criteria · Pathology $\cdot$ WHO classification · Breast Cancer}

\section{Summary}

The WHO Classification of Tumors of the Breast, 4th edition, is an update to the 3rd edition that was published in 2003, and covers all neoplastic and preneoplastic lesions of the breast. Changes to the 4th edition include new aspects and changes to the terminology that reflect our present-day knowledge of these lesions. Definitions for histopathological diagnosis are complemented by a description of clinical features, epidemiology, macroscopy, genetics, and prognostic and predictive features. In this review of the WHO classification, we have focused on invasive carcinomas, precursor lesions, and some benign epithelial proliferations.

\section{Introduction}

The WHO series of monographs on tumor classification, published by the International Agency for Research on Cancer (IARC), covers all organ systems, and continues the tradition of the WHO Blue Books series. These monographs have traditionally defined the standards on tumor classification and nomenclature for pathologists worldwide. The 4th edition of the WHO Classification of Tumors of the Breast [1] is the most recent addition to this series, and provides a timely update to many new aspects of breast cancer classification that have occurred since the publication of the 3rd edition in 2003 [2].

There has been much progress in our understanding of the pathology and molecular biology of breast cancer in the last few years. Despite this, we have not yet arrived at a molecu-

\author{
Schlüsselwörter \\ Diagnostische Kriterien · Pathologie - WHO-Klassifikation • \\ Brustkrebs
}

\section{Zusammenfassung}

Die vierte Auflage der WHO Klassifikation der Tumoren der Mamma ist ein Update der 2003 erschienenen dritten Auflage und behandelt alle neoplastischen und präneoplastischen Läsionen der Mamma. Die Änderungen der vierten Auflage betreffen neue Aspekte und Änderungen der Terminologie, und spiegeln unser gegenwärtiges Verständnis dieser Läsionen wider. Die histopathologischen Definitionen werden ergänzt um Beschreibungen der klinischen Charakteristika, der Makroskopie, Genetik und prognostischer und prädiktiver Informationen. In dieser Übersicht wird ein Überblick über die invasiven Karzinome, die Vorläuferläsionen und einige benigne Epithelproliferaionen gegeben.

larly defined classification of tumors of the breast. Therefore, the authors focused on updating the traditional tumor classification, incorporating molecular and genetic data into the morphologically defined system. This WHO tumor classification of the breast covers not only invasive breast cancers, but also precursor lesions, lesions of low malignant potential, benign epithelial proliferations, fibroepithelial, myoepithelial and mesenchymal neoplasms, among others. Its encyclopedic character makes it an essential reference for pathologists, clinicians, and researchers on breast cancers alike. Here we discuss invasive carcinomas, precursor lesions, and ductal proliferations, including papillary lesions. The book contains many important details that are of practical relevance, and an extensive list of literature. Therefore, all breast surgeons, breast oncologists, and breast pathologists are encouraged to purchase a copy.

\begin{tabular}{ll}
\hline KARGER & $\begin{array}{l}\text { ( ) 2013 S. Karger GmbH, Freiburg } \\
1661-3791 / 13 / 0082-0149 \$ 38.00 / 0\end{array}$ \\
$\begin{array}{l}\text { Fax +49 761 452 0714 } \\
\text { Information@Karger.com } \\
\text { www.karger.com }\end{array}$ & $\begin{array}{l}\text { Accessible online at: } \\
\text { www.karger.com/brc }\end{array}$
\end{tabular}

Prof. Dr. Hans-Peter Sinn

Sektion für Gynäkologische Pathologie

Pathologisches Institut

Ruprecht-Karls-Universität Heidelberg

Im Neuenheimer Feld 224, 69120 Heidelberg, Germany

peter.sinn@med.uni-heidelberg.de 
Invasive Breast Carcinoma of No Special Type (NST), Previously Known as Invasive Ductal Carcinoma (NOS)

The terminology for the most common type of breast cancer has changed from invasive ductal carcinoma, not otherwise specified (NOS) (2003) to invasive carcinoma of no special type (NST) (2012). This group of breast cancers comprises all tumors without the specific differentiating features that characterize the other categories of breast cancers. The 2012 definition of invasive carcinomas (NST) is identical to the 2003 definition of invasive ductal carcinoma (NOS), except that the name 'ductal' has been omitted in the new terminology. The rationale for this is that the term 'ductal' conveys unproven histogenetic assumptions (derivation of the tumors from the ductal system) and that invasive ductal carcinoma (NOS) does not comprise a uniform group of carcinomas. In addition, other specific types of breast cancer are also frequently associated with ductal carcinoma in situ (DCIS), and can, therefore, also be regarded as invasive ductal carcinomas, albeit of special type. Thus, the term 'ductal' does not represent a distinguishing pathological feature for breast cancers of no specific or of specific type, and was considered meaningless. The terms 'invasive ductal carcinoma' or 'ductal NOS' are accepted as alternative terminology options, but the use of 'carcinoma of no special type' is the preferred term. The diagnosis is made by exclusion of recognized specific types of breast cancers.

Carcinomas of mixed type have a specialized pattern in at least $50 \%$ of the tumor and a non-specialized pattern in between $10 \%$ and $49 \%$. These tumors are designated as mixed invasive NST and special type or mixed invasive NST and lobular carcinoma. Rare morphological variants of invasive carcinoma NST include pleomorphic carcinoma, carcinoma with osteoclast-like stromal giant cells, carcinoma with choriocarcinomatous features, and carcinoma with melanotic features.

\section{Special Subtypes of Invasive Breast Carcinoma}

The most common specific subtypes include invasive lobular, tubular, cribriform, metaplastic, apocrine, mucinous, papillary, and micropapillary carcinoma, as well as carcinoma with medullary, neuroendocrine, and salivary gland/skin adnexal type features. These specific tumor types are defined by their morphology, but are also linked to particular clinical, epidemiological, and molecular features.

With invasive lobular carcinoma (ILC), variants such as solid, alveolar, pleomorphic, tubulolobular, and mixed variants are recognized and related to differences in prognosis when compared to ILC of classic type. Among pleomorphic lobular carcinomas, apocrine, histiocytic, or signet-ring cell differentiation can be observed. Tumor grading of ILC is advocated, with the majority (approximately $76 \%$ ) of classic ILCs being grade 2 in the Nottingham histological grading system, and ILC of grade 3 comprising mostly a solid and pleomorphic subtype [3, 4]. Immunostaining with E-cadherin can help in distinguishing ILC from NST carcinomas, but as about $15 \%$ of ILCs also express E-cadherin (e.g. the tubulolobular variant), positive E-cadherin immunostaining should not be used to reclassify an ILC as an NST carcinoma.

Tubular carcinoma and invasive cribriform carcinoma are carcinomas with a particularly favorable prognosis and similar low-grade tumor nuclear features. The new WHO classification stresses the strict diagnostic requirements for these tumor types with their characteristic features being present in $>90 \%$ of the tumor. In addition, the possible clinical and therapeutic implications for invasive tubular carcinoma regarding the extent of surgery, axillary clearance, and postoperative radiotherapy are discussed.

Carcinomas with medullary features are an overlapping group of tumors with more or less 'medullary' appearance, and are described in a separate chapter of the new WHO classification. The authors advocate abandoning the terms medullary carcinoma, atypical medullary carcinoma and invasive carcinoma NST with medullary features, and recommend using the term carcinoma with medullary features for this group of tumors because of the overlapping morphological and immunohistological features and low interobserver reproducibility. The relatively good outcome of carcinomas with medullary features is attributed to the presence of a prominent lympho-plasmocytic infiltrate [5], and the presence of a B cell/plasma cell metagene in these tumors [6]. Carcinomas with medullary features are more common in patients with BRCA1 germ-line mutations.

Metaplastic carcinoma represents a group of unrelated invasive breast cancers displaying differentiation of the tumor cells into squamous or mesenchymal-looking elements. This includes, but is not limited to, spindle, chondroid, osseous, and rhabdomyoid cells, and these elements may be mixed with carcinoma of usual type. Depending on their cellular features, metaplastic carcinomas may be either low-grade tumors (e.g. low-grade adenosquamous carcinoma or low-grade spindle cell carcinoma), or high-grade tumors (e.g. high-grade squamous cell carcinoma, or high-grade spindle cell carcinoma). Because of the heterogeneity of this group of tumors, a descriptive classification is advocated. As a group, metaplastic carcinomas typically are triple-negative tumors, but have worse prognosis than other forms of triple-negative breast cancers [7].

Carcinomas with apocrine differentiation mostly comprise tumors that are otherwise of no specific type; however, apocrine differentiation is also seen in special-type carcinomas, such as lobular, micropapillary, papillary, and medullary tumors [8]. The use of the term apocrine carcinoma is, therefore, discouraged, and tumors with apocrine differentiation should be classified according to their primary invasive type. These tumors are frequently negative for estrogen and progesterone receptors, and mostly, but not always, show andro- 
gen receptor expression and/or HER2 positivity [9]. Therefore, histologically defined apocrine differentiation overlaps with, but is not identical to, the molecular apocrine subtype of breast cancer [10].

Adenoid cystic carcinoma is the most frequently encountered salivary-type tumor of the breast and is, in the great majority of cases, a low-grade malignant tumor [11]. Recently, a recurrent chromosomal translocation $\mathrm{t}(6 ; 9)$ (q22-23;p23-24), which leads to the formation of the MYB-NFIB fusion gene, has been described in adenoid cystic carcinoma [12]. Like other salivary-type tumors of the breast, it rarely spreads to the regional lymph nodes. Its characteristic histological and immunohistological features clearly distinguish this tumor from other types of invasive breast cancers. Variants, such as the solid variant with basaloid features, should be recognized, but a special grading system of adenoid cystic carcinoma has no practical value.

Mucinous carcinomas and carcinomas with signet-ring cell differentiation are described together in the new WHO classification. Carcinomas with signet-ring cell differentiation are listed among the invasive breast carcinomas (table 1), but are not regarded to represent a tumor type of its own. Rather, signet-ring cell differentiation may be observed either with invasive lobular carcinomas (mostly), where it is 1 pattern seen with the pleomorphic variant or with invasive carcinomas NST, and, rarely, also with high-grade mucinous carcinomas that otherwise show predominantly extracellular mucin production. No specific prognosis is believed to be associated with signet-ring cell differentiation.

Invasive mucinous carcinoma of the breast characteristically has a luminal mucinous A molecular subtype [10], but mucinous carcinomas presenting with large cell clusters as hypercellular or type B mucinous carcinomas [13] show a gene expression pattern that is similar to that of neuroendocrine carcinomas [14]. Prognostically, pure mucinous carcinomas have an excellent prognosis, but the outcome for mixed mucinous carcinomas is similar to NST tumors [15].

Carcinomas with neuroendocrine features are, to the most part, tumors with low- or intermediate-grade nuclear features, and morphological features similar to those of neuroendocrine tumors of the gastrointestinal tract and of the lung. The appropriate term for these tumors is neuroendocrine tumor, well-differentiated. In addition, NST carcinomas, and some special variants of mucinous and papillary carcinomas may exhibit neuroendocrine features; these are summarized as invasive breast carcinoma with neuroendocrine differentiation. Rarely, poorly differentiated neuroendocrine/small cell carcinomas are encountered. The neuroendocrine differentiation correlates with an expression of estrogen and progesterone receptors in the majority of the well- and moderately differentiated tumors and in $>50 \%$ of poorly differentiated/small cell carcinomas. Prognosis for tumors with neuroendocrine differentiation is related to conventional histological tumor grading and staging, but not to the neuroendocrine differentiation
Table 1. Invasive breast carcinomas (without microinvasive carcinoma and invasive papillary lesions)

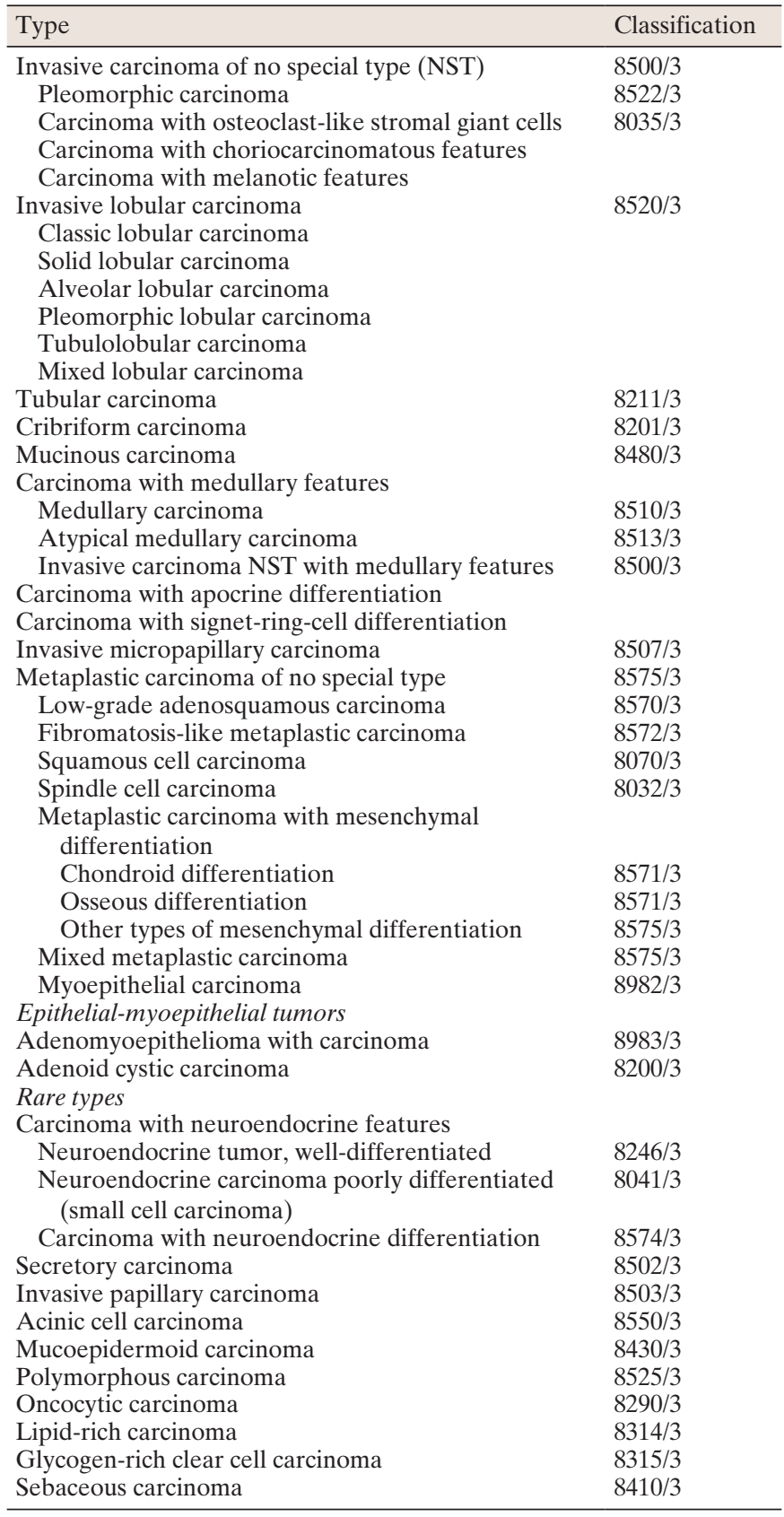

per se [16]. However, in selected series of neuroendocrine carcinomas of the breast, the prognosis was reported as either better [17] or worse [18] than that in control patients.

Invasive papillary carcinoma of the breast is regarded as a specially differentiated adenocarcinoma of the breast with papillary morphology, but otherwise no distinguishing clinical, genetic, or prognostic features. Invasive micropapillary carcinoma is a luminal-type breast cancer with a propensity for lymphovascular invasion and regional lymph-node metastasis. The possible relationship of this feature with an impaired prognosis for the patient remains to be determined; 1 report, however, has confirmed this association [19]. 
Exceptionally rare tumor types and variants include secretory carcinoma of the breast - a tumor with a distinctive genetic translocation occurring in young patients. Oncocytic carcinoma resembles oncocytic tumors of the kidney and thyroid. Other rare tumor types include polymorphous carcinoma, sebaceous carcinoma of the breast, lipid-rich carcinoma, glycogen-rich clear cell carcinoma, and acinic cell carcinoma.

\section{Special Situations}

Microinvasive carcinoma is a subset of pT1 breast cancer, in which cancer cells infiltrate beyond the basement membrane at 1 or more foci, but none measuring more than $0.1 \mathrm{~cm}$ in greatest dimension. The new WHO classification has adopted the TNM definition of the UICC/AJCC for microinvasion [20]. Microinvasive carcinoma is usually seen in cases with extensive DCIS, notably of high nuclear grade. The application of strict histological criteria for the diagnosis of microinvasion is stressed to avoid overdiagnosis, which may significantly overestimate its incidence [21]. Because of varying diagnostic criteria for microinvasion, no robust data on the clinical behavior of microinvasive carcinoma are available, but the authors express the opinion that patient prognosis is excellent and not clearly different from patients with pure DCIS of equivalent size and grade. It is recommended that the therapy for microinvasive carcinoma follows the same principles as that for high-grade DCIS.

Inflammatory carcinoma is a very aggressive form of breast carcinoma with distinctive clinical and/or pathological criteria [22]. Clinical symptoms include rapid breast enlargement, and skin changes (redness, edema, peau d' orange) involving more than a third of the breast. Often the breast does not have a discrete palpable mass, but a diffuse firmness is common. The pathological equivalent to the clinical signs and symptoms is the presence of numerous lymphatic tumor cell emboli in the breast skin. A diagnosis of inflammatory carcinoma can be made if either the typical clinical and/or the characteristic histopathological features are present. The immunoprofile of the tumors is variable, but most are NST carcinomas, grade 3 .

With bilateral breast cancer, synchronous and non-synchronous bilateral breast carcinoma need to be distinguished. Synchronous breast cancer is diagnosed when a contralateral breast carcinoma occurs simultaneously or within 3 months of the primary, whereas metachronous bilateral breast cancer is considered when it occurs more than 3 months after diagnosis of the first tumor [23]. However, from an epidemiological standpoint, an interval of 12 months might be considered more appropriate [24]. Bilateral breast cancer needs to be distinguished from metastatic cancer in the contralateral breast, which is a much rarer situation. To differentiate metastatic cancer from bilaterality, several aspects have to be considered, including tumor type, grade, presence of intraductal carcinoma, lymphatic emboli, and the lymph node status.

\section{Precancerous Lesions}

The new WHO classification lists both ductal carcinoma in situ (DCIS) and lobular neoplasia as precursor lesions of the breast, but emphasizes their different clinical behavior and the consequential differences in therapeutic recommendations, which have to be based on the biology of the disease. Therefore, both lesions have to be clearly distinguished pathologically.

Lobular neoplasia is subgrouped into classic lobular carcinoma in situ (LCIS) and pleomorphic lobular carcinoma in situ (PLCIS). In accordance with the 3rd edition of the International classification of diseases for oncology (ICD-O-3) [25], both lesions are assigned the behavior code $/ 2$ (in situ), but are distinguished from atypical lobular hyperplasia $(\mathrm{ALH})$, which is also grouped among the lobular neoplasias, but not assigned a behavior code (table 2). The distinction of ALH from LCIS is based on the extent of involvement of individual lobular units [26], and classic LCIS is diagnosed when a distension of more than half of the acini of a lobular unit by a uniform and discohesive population of small atypical epithelial cells is observed. Variants of classic LCIS include forms with marked distension and comedo-type necrosis, as well as pleomorphic and apocrine pleomorphic LCIS. The clinical significance of these variants, and the appropriate management is uncertain at the present time, but it is stressed that LCIS has a significantly higher risk of subsequent invasive cancer than ALH. Specific histological features, including the degree of pleomorphism, bulk of disease, solid-duct involvement and the presence of comedo necroses are mentioned to distinguish potentially more aggressive forms of LCIS that would merit consideration for complete excision, but the existing evidence to support this is cited to be 'lim-

Table 2. Precursor lesions, intraductal proliferative lesions, and papillary lesions

\begin{tabular}{ll}
\hline Type & Classification \\
\hline Precursor lesions & \\
$\quad$ Ductal carcinoma in situ & $8500 / 2$ \\
$\quad$ Lobular neoplasia & \\
$\quad$ Lobular carcinoma in situ & $8520 / 2$ \\
$\quad$ Classic lobular carcinoma in situ & $8519 / 2^{*}$ \\
$\quad$ Pleomorphic lobular carcinoma in situ & \\
$\quad$ Atypical lobular hyperplasia & \\
Intraductal proliferative lesions & \\
$\quad$ Usual ductal hyperplasia & \\
$\quad$ olumnar cell lesions including flat epithelial atypia & \\
Atypical ductal hyperplasia & \\
Papillary lesions & $8503 / 0$ \\
Intraductal papilloma & $8503 / 0$ \\
$\quad$ Intraductal papilloma with atypical hyperplasia & $8503 / 2 *$ \\
$\quad$ Intraductal papilloma with ductal carcinoma & \\
$\quad$ in situ & $8520 / 2$ \\
$\quad$ Intraductal papilloma with lobular carcinoma & \\
$\quad$ in situ & $8503 / 2$ \\
Intraductal papillary carcinoma & $8504 / 2$ \\
Encapsulated papillary carcinoma & $8504 / 3$ \\
$\quad$ Encapsulated papillary carcinoma with invasion & \\
Solid papillary carcinoma & $8509 / 2$ \\
$\quad$ In situ & $8509 / 3$ \\
$\quad$ Invasive &
\end{tabular}


ited'. Therefore, no recommendation is made for complete excision of either LCIS or pleomorphic LCIS, but the treatment should be based upon the individual situation.

DCIS is characterized as having an inherent, but not necessarily obligate tendency for progression to invasive breast cancer. The proposed grading of DCIS is based upon its nuclear features, and, in contrast to the preceding WHO classification, the diagnosis of DCIS of low nuclear grade is not precluded by the presence of focal punctate or comedotype necrosis. Comedo-type necrosis is commonly seen with high-grade DCIS, but is not obligatory. The traditional classification, which is based on its pattern, should also be reported, as well as the presence and type of necrosis, cell polarization, the size (extent) of the lesion, the location of associated microcalcifications (with DCIS, with benign tissue, or both), and the status of the surgical margins [27].

\section{Intraductal Proliferative Lesions}

Whereas DCIS is regarded as a true precursor lesion of invasive breast cancer, intraductal proliferative lesions comprise a group of cytologically and architecturally diverse proliferations originating in the terminal ducto-locular unit (TDLU) and are associated with an increased risk (of varying magnitude) for the subsequent development of invasive breast cancer. The classification of intraductal proliferative lesions is under debate. This includes the distinction of atypical ductal hyperplasia (ADH) from low-grade DCIS, and the use of the ductal intraepithelial neoplasia (DIN) system. The use of this terminology is discouraged, in part because it provides no new diagnostic criteria, and so does not improve the interobserver variability in the diagnosis of intraductal proliferative lesions.

\section{Usual Ductal Hyperplasia}

The term usual ductal hyperplasia (UDH, formerly called epitheliosis) is now used to distinguish this lesion in its common and florid forms from ADH. Well-known histological and immunohistological criteria are mentioned in this context. UDH carries a slightly increased risk (about 1.5-2 fold) for breast cancer.

\section{Columnar Cell Lesions}

These changes of the TDLUs, which frequently are accompanied by intraluminal secretions and microcalcifications, also carry a very low risk for the subsequent development of breast cancer (relative risk about 1.5). Columnar cell change and columnar cell hyperplasia may be associated with other types of benign epithelial proliferative lesions, and are distinguished from higher risk lesions by the lack of cellular atypia.

\section{Flat Epithelial Atypia}

This lesion is regarded as the neoplastic counterpart of benign columnar cell lesions, and is also associated with intraluminal secretion and microcalcifications that point to the diagnosis of flat epithelial atypia (FEA) radiologically. FEA is characterized by the presence of 1 or several layers of cuboidal to columnar cells that lack polarity and show round, uniform nuclei and inconspicuous nucleoli. While occasional cellular tufts or mounds may occur, other well-developed architectural disturbances (micropapillae, arcades, bridges) are absent. The clinical management of these low-risk lesions rests upon careful radiological-pathological correlation.

\section{Atypical Ductal Hyperplasia}

Among the intraductal proliferative lesions, ADH carries the highest probability for the subsequent risk of invasive breast cancer (relative risk about 3.0-5.0) [28]. The morphological features of $\mathrm{ADH}$ are identical to those of low-grade DCIS, but $\mathrm{ADH}$ is limited in size. There are 2 quantitative criteria that distinguish ADH from low-grade DCIS: the presence of homogeneous involvement of not more than 2 membranebound spaces; or a size of $\leq 2 \mathrm{~mm}$. The use of 1 or both criteria is considered appropriate by the authors of the WHO classification. In core needle biopsies, the term 'atypical intraductal proliferative lesion' may alternatively be used to prompt a surgical excision, and the definitive diagnosis may be deferred to the evaluation of the surgical specimen.

\section{Papillary Lesions}

The benign intraductal papilloma occurs as a central papilloma originating from the ducts in the subareolar region or peripherally, and in both locations can be either solitary or multiple. Both central and peripheral papillomas are characterized by fibrovascular cores with epithelial and myoepithelial cell layers. Central intraductal papillomas with a predominant or exclusive glandular differentiation are called ductal adenoma. Intraductal papillomas and ductal adenomas may show regressive changes such as sclerosis or infarction, as well as epithelial or myoepithelial hyperplasia or squamous or apocrine metaplasia. These changes may cause diagnostic difficulties in core needle biopsy [29]. The term papillomatosis is not used in the WHO classification of the breast, because historically this was used both for usual-type ductal hyperplasia and for papillomas.

Atypical epithelial proliferations (ADH and DCIS) may occur in papillomas, and are usually of low grade. As with atypical intraductal proliferative lesions, the distinction of $\mathrm{ADH}$ and DCIS within a papilloma relies on quantitative criteria. An intraductal papilloma with $A D H$ is diagnosed when the atypical epithelial proliferation is $<3 \mathrm{~mm}$, while larger atypical epithelial proliferations within a papilloma fulfill the criteria of an intraductal papilloma with low-grade DCIS [30]. This definition replaces alternative terminologies that were focused on the proportion of atypical cells (30 or 90\%) within a papilloma. An intermediate- or high-grade DCIS within a papilloma can be diagnosed regardless of the extent of atypia. 
Intraductal papillary carcinoma is one of the architectural patterns of DCIS and must be clearly differentiated from benign or atypical papillomas. It may occur together with other patterns of DCIS, such as micropapillary and cribriform patterns, but comedo-type necrosis is uncommon with intraductal papillary carcinoma. Histologically, intraductal papillary carcinoma is usually unrelated to the presence of intraductal papilloma(s), and is not thought to arise from papillomas.

The diagnostic term for larger intracystic papillary carcinomas has changed to encapsulated papillary carcinoma because the fibrous capsule that surrounds these lesions usually lacks a myoepithelial cell layer [31]. The question of whether an encapsulated papillary carcinoma represents an in situ lesion or an indolent form of invasive papillary carcinoma has not been resolved [32]. However, the diagnosis of frankly invasive carcinoma can only be made if invasion is present outside the fibrous capsule. If not, encapsulated papillary carcinoma should be staged as papillary carcinoma in situ, and has an excellent prognosis. Regional lymph-node metastases occur only rarely without evidence of invasion.

Another form of sharply circumscribed, and frequently non-invasive papillary carcinoma is solid papillary carcinoma, but, in contrast to encapsulated papillary carcinoma, this tumor is usually composed of multiple adjacent nodules, and the papillary nature may not be readily apparent because of its predominantly solid growth patterns. Diagnostic criteria include the presence of small, monotonous cells with hyperchromatic nuclei that may show neuroendocrine or mucinous differentiation [33]. Generally, the prognosis is good, and when a surrounding myoepithelial cell layer is present, the tumor is classified as an in situ carcinoma.

\section{Disclosure Statement}

The authors declare that they have no competing interests.

\section{References}

1 Lakhani S, Ellis I, Schnitt S, et al.: WHO Classification of Tumours of the Breast, 4th ed. Lyon, IARC Press, 2012.

2 Tavassoli FA, Devilee P (eds): Pathology and Genetics of Tumours of the Breast and Female Genital Organs. Lyon, IARC Press, 2003.

$\checkmark 3$ Orvieto E, Maiorano E, Bottiglieri L, et al.: Clinicopathologic characteristics of invasive lobular carcinoma of the breast: results of an analysis of 530 cases from a single institution. Cancer 2008;113:1511-1520.

4 Talman ML, Jensen MB, Rank F: Invasive lobular breast cancer. Prognostic significance of histological malignancy grading. Acta Oncol 2007;46:803809.

5 Rakha EA, Aleskandarany M, El-Sayed ME, et al.: The prognostic significance of inflammation and medullary histological type in invasive carcinoma of the breast. Eur J Cancer 2009;45:1780-1787.

6 Rody A, Karn T, Liedtke C, et al.: A clinically relevant gene signature in triple negative and basal-like breast cancer. Breast Cancer Res 2011;13:R97.

7 Lee H, Jung SY, Ro JY, et al.: Metaplastic breast cancer: Clinicopathological features and its prognosis. J Clin Pathol 2012;65:441-446.

$>8$ Abati AD, Kimmel M, Rosen PP: Apocrine mammary carcinoma. A clinicopathologic study of 72 cases. Am J Clin Pathol 1990;94:371-377.

$\checkmark 9$ Niemeier LA, Dabbs DJ, Beriwal S, et al.: Androgen receptor in breast cancer: Expression in estrogen receptor-positive tumors and in estrogen receptor-negative tumors with apocrine differentiation. Mod Pathol 2010;23:205-212.

10 Weigelt B, Horlings HM, Kreike B, et al.: Refinement of breast cancer classification by molecular characterization of histological special types. J Pathol 2008;216:141-150.

11 Foschini MP, Krausz T: Salivary gland-type tumors of the breast: A spectrum of benign and malignant tumors including 'triple negative carcinomas' of low malignant potential. Semin Diagn Pathol 2010;27:77-90.

12 Wetterskog D, Lopez-Garcia MA, Lambros MB, et al.: Adenoid cystic carcinomas constitute a genomically distinct subgroup of triple-negative and basal-like breast cancers. J Pathol 2012;226:84-96.

13 Capella C, Eusebi V, Mann B, et al.: Endocrine differentiation in mucoid carcinoma of the breast. Histopathology 1980;4:613-630.

14 Lacroix-Triki M, Suarez PH, MacKay A, et al.: Mucinous carcinoma of the breast is genomically distinct from invasive ductal carcinomas of no special type. J Pathol 2010;222:282-298.

15 Toikkanen S, Kujari H: Pure and mixed mucinous carcinomas of the breast: A clinicopathologic analysis of 61 cases with long-term follow-up. Hum Pathol 1989;20:758-764.

16 Miremadi A, Pinder S, Lee A, et al.: Neuroendocrine differentiation and prognosis in breast adenocarcinoma. Histopathology 2002;40:215-222.

17 Lopez-Bonet E, Alonso-Ruano M, Barraza G, et al.: Solid neuroendocrine breast carcinomas: Incidence, clinico-pathological features and immunohistochemical profiling. Oncol Rep 2008;20:13691374.

18 Wei B, Ding T, Xing Y, et al.: Invasive neuroendocrine carcinoma of the breast: A distinctive subtype of aggressive mammary carcinoma. Cancer 2010;116:4463-4473.

19 Chen L, Fan Y, Lang RG, et al.: Breast carcinoma with micropapillary features: Clinicopathologic study and long-term follow-up of 100 cases. Int J Surg Pathol 2008;16:155-163.

20 American Joint Committee on Cancer: AJCC cancer staging manual, 7th ed. New York, Springer, 2010.

21 Prasad ML, Osborne MP, Giri DD, et al.: Microinvasive carcinoma (T1mic) of the breast: clinicopathologic profile of 21 cases. Am J Surg Pathol 2000;24:422-428.
22 Lucas FV, Perez-Mesa C: Inflammatory carcinoma of the breast. Cancer 1978;41:1595-1605.

23 Hartman M, Czene K, Reilly M, et al.: Incidence and prognosis of synchronous and metachronous bilateral breast cancer. J Clin Oncol 2007;25:4210 4216.

24 Huo D, Melkonian S, Rathouz PJ, et al.: Concordance in histological and biological parameters between first and second primary breast cancers. Cancer 2011;117:907-915.

25 Fritz AG (ed): International Classification of Diseases for Oncology: ICD-O, 3rd ed. Geneva World Health Organization, 2000

26 Page DL, Dupont WD, Rogers LW, et al.: Atypical hyperplastic lesions of the female breast. A longterm follow-up study. Cancer 1985;55:2698-2708.

27 The Consensus Committee: Consensus conference on the classification of ductal carcinoma in situ. Hum Pathol 1997;28:1221-1225.

28 Fitzgibbons PL, Henson DE, Hutter RVP: Benign breast changes and the risk for subsequent breast cancer - An update of the 1985 consensus statement. Arch Pathol Lab Med 1998;122:1053-1055.

29 Bilous M: Breast core needle biopsy: Issues and controversies. Mod Pathol 2010;23 Suppl 2:S36-45.

30 Page DL, Salhany KE, Jensen RA, et al.: Subsequent breast carcinoma risk after biopsy with atypia in a breast papilloma. Cancer 1996;78:258-266.

31 Collins LC, Carlo VP, Hwang H, et al.: Intracystic papillary carcinomas of the breast: A reevaluation using a panel of myoepithelial cell markers. Am J Surg Pathol 2006;30:1002-1007.

32 Hill CB, Yeh IT: Myoepithelial cell staining patterns of papillary breast lesions: From intraductal papillomas to invasive papillary carcinomas. Am J Clin Pathol 2005;123:36-44.

33 Nassar H, Qureshi H, Volkanadsay N, et al.: Clinicopathologic analysis of solid papillary carcinoma of the breast and associated invasive carcinomas. Am J Surg Pathol 2006;30:501-507. 